\title{
Hyposalivation and 10-year all-cause mortality in an elderly Japanese population
}

\author{
Masanori Iwasaki $^{1,2}$ (D) | Wenche S. Borgnakke ${ }^{3}$ (D) | Akihiro Yoshihara4 (D) | \\ Kayoko Ito ${ }^{5}$ (D) | Hiroshi Ogawa ${ }^{1}$ | Kaname Nohno ${ }^{1}$ | Misuzu Sato ${ }^{1}$ | \\ Kumiko Minagawa $^{1}$ | Toshihiro Ansai ${ }^{2}$ | Hideo Miyazaki ${ }^{1}$
}

${ }^{1}$ Division of Preventive Dentistry, Department of Oral Health Science, Niigata University Graduate School of Medical and Dental Sciences, Niigata, Japan

${ }^{2}$ Division of Community, Oral Health Development, Kyushu Dental University, Kitakyushu, Japan

${ }^{3}$ Department of Periodontics and Oral Medicine, University of Michigan School of Dentistry, Ann Arbor, Michigan, USA

${ }^{4}$ Division of Oral Science for Health Promotion, Department of Oral Health and Welfare, Niigata University Graduate School of Medical and Dental Sciences, Niigata, Japan

${ }^{5}$ Oral Rehabilitation, Niigata University Medical and Dental Hospital, Niigata, Japan

\section{Correspondence}

Masanori Iwasaki, Division of Community Oral Health Development, Kyushu Dental University, Kitakyushu, Japan.

Email: r14iwasaki@fa.kyu-dent.ac.jp

\section{Funding information}

This work was supported by a Grant-in-Aid from the Ministry of Health and Welfare of Japan (H10-Iryo-001, H13-Iryo-001 and H16Iryo-020) and JSPS KAKENHI (26861827).
Objective: To evaluate the association of salivary flow rate with all-cause mortality among older Japanese adults. We hypothesised that hyposalivation would be a marker for mortality.

Background: Hyposalivation, which is an objectively measurable decrease in salivary flow, is highly prevalent among older adults. It is associated with malnutrition and poor general health.

Methods: The study population comprised 600 community-dwelling Japanese adults (306 men and 294 women), who were 70 years old at baseline. They underwent stimulated salivary flow rate (SSFR) measurements and were followed up during a 10-year study period. After stratification by sex, the hazard ratios of all-cause mortality were estimated using Cox proportional hazards regression analysis comparing groups with and without hyposalivation (ie, SSFR $<0.7 \mathrm{~mL} / \mathrm{min}$ ).

Results: The baseline prevalence of hyposalivation was $27.8 \%$ (85/306) among men and $47.3 \%$ (139/294) among women. During a mean (standard deviation) follow-up period of 104 (27) months, 80 deaths occurred: 60 (75.0\%) deaths among men and 20 (25.0\%) deaths among women. After adjusting for the number of remaining teeth, smoking status, exercise, hypoalbuminemia, diabetes and cardiovascular disease, hyposalivation at baseline was significantly associated with all-cause mortality among men (adjusted hazard ratio, 1.71; 95\% confidence interval, 1.01-2.89). In contrast, no association between SSFR and all-cause mortality existed among women.

Conclusion: Hyposalivation could be a marker for all-cause mortality among older community-dwelling Japanese men. Future studies investigating the association between SSFR and cause-specific mortality are warranted.

KEYWORDS

aged, cohort studies, epidemiology, saliva
The addresses of the institutions at which the work was carried out: Division of Preventive Dentistry, Department of Oral Health Science, Niigata University Graduate School of Medical and Dental Sciences, 2-5274 Gakkocho-Dori, Chuo-Ku, Niigata 951-8514, Japan.

\section{1 | INTRODUCTION}

Hyposalivation, which is an objectively measurable decrease in salivary flow, is highly prevalent among older adults. ${ }^{1-3}$ A 2015 meta-analysis showed that salivary flow decreases with age. ${ }^{4}$ Saliva has a major role 
in oral functions such as chewing, tasting and swallowing. ${ }^{5}$ Therefore, hyposalivation can deteriorate oral function ${ }^{6}$ and prevent adequate nutritional intake. ${ }^{7}$ Previous studies have shown that a decreased salivary flow rate was associated with a low nutritional assessment score, ${ }^{8}$ as well as undernutrition as determined by arm circumference, triceps skinfold thickness and low serum albumin concentration. ${ }^{9,10}$

In addition, owing to its mechanical clearance and antimicrobial properties, saliva has an important role in inhibiting bacterial adhesion to mucosal tissues and bacterial colonisation. ${ }^{5}$ Therefore, decreased salivary output can increase susceptibility to bacterial colonisation in the oral cavity and the upper respiratory tract, which may lead to respiratory infections, especially in elderly individuals. ${ }^{11-13}$

Given the age-related decrease in salivary flow and the possible deterioration in nutritional status and general health, there is a possibility that hyposalivation is a marker for mortality in older adults. Therefore, this longitudinal study was designed to evaluate the potential association of salivary flow rate with mortality from any cause among older Japanese adults.

\section{2 | METHODS}

\section{1 | Study design and population}

This study was designed as a 10 -year prospective cohort study. The study population consisted of 600 randomly selected men and women who resided in Niigata City, Japan. They were members of the Niigata Study, an epidemiological study investigating the effects of oral health and function on general health. All participants were aged 70 years at study enrolment (June 1998). Details of the sampling method have previously been described. ${ }^{14}$ After the participants provided written informed consent, they underwent baseline examinations. The study population was then followed up until the date of death, the date of disenrollment, the date of leaving Niigata city or the end of follow-up (June 2008), whichever occurred first. This study was performed in accordance with the Declaration of Helsinki and was approved by the Ethics Committee of the Niigata University Faculty of Dentistry (approval number: 12-R1-4-21).

\section{2 | Baseline examination}

Stimulated whole saliva was collected between 9:00 AM and 3:00 PM by a single trained dentist, based on a previously published saliva sample collection protocol. ${ }^{15}$ In brief, participants were asked to chew $1 \mathrm{~g}$ of tasteless paraffin wax for 3 minutes and to expectorate secreted saliva into a test tube. During saliva collection, any participants who wore dentures kept them in place. The absolute amount of stimulated saliva was measured in millilitres, and the stimulated salivary flow rate (SSFR) was calculated in millilitres per minute. Based on the criterion used in previous studies, hyposalivation was defined as an SSFR $<0.7 \mathrm{~mL} / \mathrm{min}^{1,16}$

Four trained dentists determined the number of retained teeth and the use of dentures and assessed the participants' periodontal status. Remaining teeth were counted as all erupted permanent teeth, except for residual roots. During the periodontal status assessment, the probing pocket depth (PPD) and bleeding on probing (BOP) were recorded at six sites for each tooth. Periodontal disease was defined as one or more sites with a PPD $\geq 4 \mathrm{~mm}$ and $\geq 10 \%$ BOP. ${ }^{17}$

Participants completed a questionnaire covering their socioeconomic status (income and level of education), health behaviour (living situation, smoking status, drinking habits and exercise habits) and medical conditions (physician diagnosis of hypertension, diabetes, cardiovascular disease [CVD] and Sjögren's syndrome). Participants were also asked whether they had received radiation therapy in the head or neck area ("yes" or "no"). In addition, participants were asked whether they took any prescribed medications ("yes" or "no"). For the participants who were taking medications (ie, answered "yes" to the above question), they were additionally asked whether they were taking two or more medications ("yes" or "no"). Use of two or more prescribed medications was defined as polypharmacy.

To obtain data on subjective oral health and function, participants were asked to answer the following "yes" or "no" questions: "Does your mouth feel dry?" "Do you have difficulty chewing?" and "Do you have difficulty speaking?" In addition, masticatory ability was assessed using a questionnaire including four types of food (French bread, rice crackers, pickled vegetables and peanuts). These four foods were considered to be hard in texture and difficult to chew. Participants who reported being able to eat $\leq 2$ of these foods were defined as having low masticatory ability. ${ }^{18}$

Height and weight were measured and used to calculate the body mass index (BMI). Fasting blood samples were collected for measuring the levels of albumin, triglycerides and total cholesterol. Hypoalbuminemia was defined as an albumin level $<4 \mathrm{~g} / \mathrm{dL}$. Dyslipidemia was defined as a total cholesterol level $\geq 220 \mathrm{mg} / \mathrm{dL}$ and/or a triglyceride level $\geq 250 \mathrm{mg} / \mathrm{dL}$.

\subsection{Statistical analyses}

All statistical analyses were performed using STATA 14.2 (StataCorp, College Station, TX, USA). The level of significance was set at $\alpha=.05$.

Because of sex differences in longevity ${ }^{19}$ and the consequent expected differences in mortality during the 10 years, all analyses were stratified by sex. Differences between groups with and without hyposalivation for the baseline characteristics were evaluated using the Mann-Whitney U-test or chi-square test, as appropriate.

All-cause mortality was the primary outcome. An initial survival analysis was performed using the Kaplan-Meier survival method, and unadjusted survival curves between groups with and without hyposalivation were compared using the log-rank test.

Univariable and multivariable Cox proportional hazards regression models were then used to assess the association between baseline hyposalivation and mortality. The proportional hazards assumption was verified by using Schoenfeld residuals. The multivariable model was developed, as follows. Each baseline variable was tested in a univariable model of the crude association with mortality. Variables that were significantly associated with mortality in the univariable analysis were considered potential confounders and subsequently entered into the multivariable model. The tested baseline variables included the number of remaining teeth (ie, the continuous variable); denture use ("yes" or "no"); periodontal disease ("yes" or "no"); annual household income 
( $\geq 2000000$ Japanese yen [JPY] or $<2000000$ JPY, which is 14419 United States dollars [USD]; in June 1998, 1 USD was 138.7 JPY); educational attainment ( $>$ or $\leq 9$ years); living situation (living alone or with others); smoking status (current smoker or previous smoker/nonsmoker); drinking habit (current drinker or nondrinker); exercise habit (involved in regular exercise [ $>30 \mathrm{~min} / \mathrm{session}$ and $\geq 2$ times/wk] or no regular exercise); polypharmacy ("yes" or "no"); activities of daily living, as assessed using the Tokyo Metropolitan Institute of Gerontology Index of Competence (TMIG-IC) index value of less than or greater than or equal to the median value; $\mathrm{BMI}$ ( $<$ or $\geq 18.5 \mathrm{~kg} / \mathrm{m}^{2}$ ); abnormalities in serum biomarkers ("yes" or "no"); hypertension ("yes" or "no"); diabetes ("yes" or "no"); and CVD ("yes" or "no").

In the sensitivity analysis, male and female participants were classified into tertile groups ("high," "middle" and "low"), based on the SSFR. The association of these SSFR tertile categories with mortality was also examined using Cox proportional hazards regression models. The dose-response relationship of all-cause mortality was analysed by trend test across the tertiles in which the SSFR tertile categories were put into the model as ordinal variables.

\section{3 | RESULTS}

Table 1 shows the study population's baseline characteristics, stratified by SSFR and sex. At baseline, no participant had received radiation therapy in the head or neck area or had been diagnosed with Sjögren's syndrome. No participant had dental implants. The mean (standard deviation [SD]) SSFRs were $1.95(0.81) \mathrm{mL} / \mathrm{min}$ in men and $1.91(0.83) \mathrm{mL} /$ min in women. Hyposalivation was observed in $27.8 \%$ (85/306) of men and $47.3 \%$ (139/294) of women. Men with hyposalivation were more likely to have periodontal disease, gingival bleeding and a history of CVD than men without hyposalivation. Polypharmacy was more likely in women with hyposalivation than those without. Self-reported difficulties in chewing and speaking were associated with hyposalivation in both sexes. Poor masticatory ability based on the number of chewable foods was borderline but not statistically significant. There was no association between self-reported oral dryness and hyposalivation.

The mean (SD) follow-up period was 104 (27) months. During the follow-up period, 80 deaths occurred: 60 (75.0\%) deaths among men and 20 (25.0\%) deaths among women. In addition, 131 participants were lost to follow-up: 123 participants withdrew from the study and eight participants left Niigata City. If participants were lost to follow up, they were censored on the date they were last seen or contacted. Compared to participants who completed follow-up, the participants who were lost to follow up were more frequently men, individuals who were less educated and individuals who were less involved in regular exercise (data not shown). Figure 1 shows the Kaplan-Meier curves of survival rates by hyposalivation. Greater all-cause mortality was observed among men with hyposalivation $(P=.01)$.

To identify potential confounders, crude associations of baseline health characteristics with mortality were studied (Table 2). In men, an increased all-cause mortality was significantly associated with smoking, hypoalbuminemia, diabetes and CVD, whereas a reduced all-cause mortality was significantly associated with the number of remaining teeth and regular exercise. In women, hypoalbuminemia and CVD were significantly associated with an increased all-cause mortality, whereas the number of remaining teeth was significantly associated with a reduced all-cause mortality. The number of female current smokers was too small to be analysed. The aforementioned variables that were significantly associated with mortality in the univariable analysis were then entered into the multivariable model.

Table 3 shows the Cox proportional hazards regression analyses for the association between hyposalivation and all-cause mortality. The univariable model revealed that hyposalivation was significantly associated with all-cause mortality among men (hazard ratio [HR], 1.94; $95 \%$ confidence interval [Cl], 1.16-3.25). This association was attenuated but remained significant after adjusting for confounders such as the number of remaining teeth, smoking status, exercise, hypoalbuminemia, diabetes and CVD (adjusted hazard ratio [aHR], 1.71; $95 \% \mathrm{Cl}$, 1.01-2.89). Sensitivity analyses also showed a significant association between SSFR and mortality among men (Table 3). After adjusting for potential confounders, the low SSFR tertile was significantly associated with all-cause mortality (vs high SSFR tertile: aHR, 2.05; $95 \% \mathrm{Cl}$, 1.01-4.17). In addition, the lower SSFR group showed a trend towards greater mortality ( $P$ for trend $=.045$ ). By contrast, the SSFR was not associated with all-cause mortality among women.

\section{4 | DISCUSSION}

This 10-year prospective cohort study found greater all-cause mortality rate in the cohort of men with hyposalivation (ie, an SSFR below the accepted threshold) at baseline.

Hyposalivation can be caused by radiation therapy, diabetes, as an adverse effect of medication, and autoimmune diseases such as Sjögren's syndrome. ${ }^{4,20}$ In the current study, the cause of hyposalivation was not investigated. The findings of the current study suggest that objective evidence of decreased salivary output, irrespective of cause, is a marker of all-cause mortality in older community-dwelling Japanese men. To the authors' knowledge, this is the first study to show this relationship.

Hyposalivation and self-reported oral dryness were not significantly related, which is in line with previous findings. ${ }^{1,3}$ Hyposalivation was found to be more prevalent in women than men. This finding concurs with a previous study that investigated the factors associated with hyposalivation among older community-dwelling people in Japan. ${ }^{3}$ Besides sex, evidence also suggests that smoking is associated with saliva secretion. There are conflicting results regarding the effects of smoking on saliva secretion. Some studies reported that smoking decreases saliva secretion while others found that smoking either increased the effect or had no effect on saliva secretion. ${ }^{1,3,21,22}$ In our study, we found no association between current smoking and hyposalivation. The observed association between hyposalivation and all-cause mortality remained significant after adjusting for smoking, suggesting this association was independent of smoking. Additionally, no statistically significant interaction was found between hyposalivation and smoking on all-cause mortality (data not shown). 
TAB LE 1 Baseline characteristics, stratified by stimulated salivary flow rate and sex

\begin{tabular}{|c|c|c|c|c|c|c|c|c|}
\hline & \multicolumn{4}{|l|}{ Men } & \multicolumn{4}{|l|}{ Women } \\
\hline & \multirow{3}{*}{$\begin{array}{l}\text { Total } \\
n=306\end{array}$} & \multirow{3}{*}{$\begin{array}{l}\text { Hyposalivation } \\
\text { (SSFR } \\
<0.7 \mathrm{~mL} / \mathrm{min} \text { ) } \\
\mathrm{n}=85\end{array}$} & $\begin{array}{l}\text { Hyposalivation } \\
\text { (SSFR }\end{array}$ & & \multirow[b]{3}{*}{$n=294$} & \multirow{3}{*}{$\begin{array}{l}\text { Hyposalivation } \\
\text { (SSFR } \\
<0.7 \mathrm{~mL} / \mathrm{min} \text { ) } \\
\mathrm{n}=139\end{array}$} & \multirow{3}{*}{$\begin{array}{l}\text { No } \\
\text { Hyposalivation } \\
\text { (SSFR } \\
\geq 0.7 \mathrm{~mL} / \mathrm{min} \text { ) } \\
\mathrm{n}=155\end{array}$} & \multirow{3}{*}{$P^{*}$} \\
\hline & & & $\geq 0.7 \mathrm{~mL} / \mathrm{min}$ ) & & & & & \\
\hline & & & $n=221$ & $P^{*}$ & & & & \\
\hline No. of teeth, median (IQR) & $20(10-26)$ & $19(12-24)$ & $21(9-27)$ & .48 & $19(10-25)$ & $18(11-25)$ & $20(9-25)$ & .90 \\
\hline Denture use, n (\%) & $174(56.9)$ & $50(58.8)$ & $124(56.1)$ & .67 & $178(60.5)$ & $92(66.2)$ & $86(55.5)$ & .06 \\
\hline Periodontal disease, $\mathrm{n}(\%)^{\mathrm{a}}$ & $60(21.4)$ & $23(29.1)$ & 37 (18.3) & .047 & $76(27.8)$ & $38(28.6)$ & $38(27.1)$ & .79 \\
\hline PPD, median (IQR) ${ }^{a}$ & $2.0(1.7-2.4)$ & $2.0(1.7-2.4)$ & $2.0(1.7-2.4)$ & .84 & $1.8(1.5-2.2)$ & $1.8(1.5-2.1)$ & $1.9(1.6-2.3)$ & .28 \\
\hline BOP, median (IQR) ${ }^{\mathrm{a}}$ & $4.2(1.4-9.1)$ & $5.6(1.9-12.5)$ & $3.9(1.2-8.2)$ & .03 & $5.3(1.8-11.1)$ & $5.0(1.9-11.1)$ & $5.6(1.3-11.1)$ & .78 \\
\hline Difficulty chewing & $78(25.5)$ & $29(34.1)$ & $49(22.2)$ & .03 & $97(33.0)$ & $54(38.9)$ & $43(27.7)$ & .04 \\
\hline Difficulty speaking & $52(17.0)$ & $22(25.9)$ & $30(13.6)$ & .01 & $47(16.0)$ & $31(22.3)$ & $16(10.3)$ & .01 \\
\hline Low masticatory ability & $53(17.3)$ & $20(23.5)$ & $33(14.9)$ & .08 & $67(22.8)$ & $38(27.3)$ & $29(18.7)$ & .08 \\
\hline Age, $y^{b}$ & 70 & 70 & 70 & - & 70 & 70 & 70 & - \\
\hline $\begin{array}{l}\text { Annual household income < } \\
2000000 \text { JPY, } n \text { (\%) }\end{array}$ & $28(9.2)$ & $11(12.9)$ & $17(7.7)$ & .15 & $58(19.7)$ & $32(23.0)$ & $26(16.8)$ & .18 \\
\hline $\begin{array}{l}\text { Length of school educa- } \\
\text { tion } \leq 9 \mathrm{y}, \mathrm{n}(\%)\end{array}$ & 125 (40.9) & $39(45.9)$ & $86(38.9)$ & .27 & $147(50.0)$ & $68(48.9)$ & $79(51.0)$ & .73 \\
\hline Living alone, n (\%) & $6(2.0)$ & $3(3.5)$ & $3(1.4)$ & .22 & $50(17.0)$ & $26(18.7)$ & $24(15.5)$ & .46 \\
\hline Current smoker, n (\%) & $104(34.0)$ & $31(36.5)$ & $73(33.0)$ & .57 & $11(3.7)$ & $5(3.6)$ & $6(3.9)$ & .90 \\
\hline $\mathrm{BMI}<18.5 \mathrm{~kg} / \mathrm{m}^{2}, \mathrm{n}(\%)$ & $31(10.1)$ & $7(8.2)$ & $24(10.9)$ & .50 & $27(9.2)$ & $15(10.8)$ & $12(7.7)$ & .37 \\
\hline \multicolumn{9}{|l|}{ Serum biomarker, n (\%) } \\
\hline Hypoalbuminemia & $26(8.5)$ & $8(9.4)$ & $18(8.1)$ & .72 & $19(6.5)$ & $11(7.9)$ & $8(5.2)$ & .34 \\
\hline Dyslipidemia & $60(19.6)$ & $20(23.5)$ & $40(18.1)$ & .28 & $143(48.6)$ & $64(46.0)$ & $79(51.0)$ & .40 \\
\hline \multicolumn{9}{|c|}{ Self-reported medical conditions, $\mathrm{n}(\%)$} \\
\hline Hypertension & $86(28.1)$ & $30(35.3)$ & $56(25.3)$ & .08 & $62(21.1)$ & $36(25.9)$ & $26(16.8)$ & .06 \\
\hline Diabetes & $18(5.9)$ & $6(7.1)$ & $12(5.4)$ & .59 & $13(4.4)$ & $9(6.5)$ & $4(2.6)$ & .11 \\
\hline CVD & $48(15.7)$ & $20(23.5)$ & $28(12.7)$ & .02 & $17(5.8)$ & $11(7.9)$ & $6(3.9)$ & .14 \\
\hline
\end{tabular}

ADL, activities of daily living; BMI, body mass index; BOP, bleeding on probing; CVD, cardiovascular disease; IQR, interquartile range; JPY, Japanese yen; PPD, probing pocket depth; T-C, total cholesterol; SSFR, stimulated salivary flow rate; TMIG-IC, Tokyo Metropolitan Institute of Gerontology Index of Competence. Dyslipidemia is the presence of hypercholesterolemia (ie, total cholesterol level $\geq 220 \mathrm{mg} / \mathrm{dL}$ ) and/or hypertriglyceridemia (ie, triglyceride level $\geq 150 \mathrm{mg}$ / $\mathrm{dL})$. Hypoalbuminemia is an albumin level $<4.0 \mathrm{~g} / \mathrm{dL}$.

Bold text indicates statistically significant associations $(P<.05)$.

${ }^{\mathrm{a}}$ The number (n)of individuals analysed is 259 men and 255 women (excluding individuals with no teeth).

${ }^{\mathrm{b}}$ All participants were $70 \mathrm{y}$ old at baseline.

${ }^{*} P$ value for the comparison between groups.

The SSFR was not significantly associated with all-cause mortality among female participants. During the decade-long follow-up period, fewer deaths occurred among women than among men: 20 deaths among women vs 60 deaths among men. National data in Japan show that women have a longer life expectancy than men. ${ }^{19}$ A potential explanation for the lack of a significant association between SSFR and mortality among female participants was that the fewer deaths among female participants influenced the predictive power. Future studies 


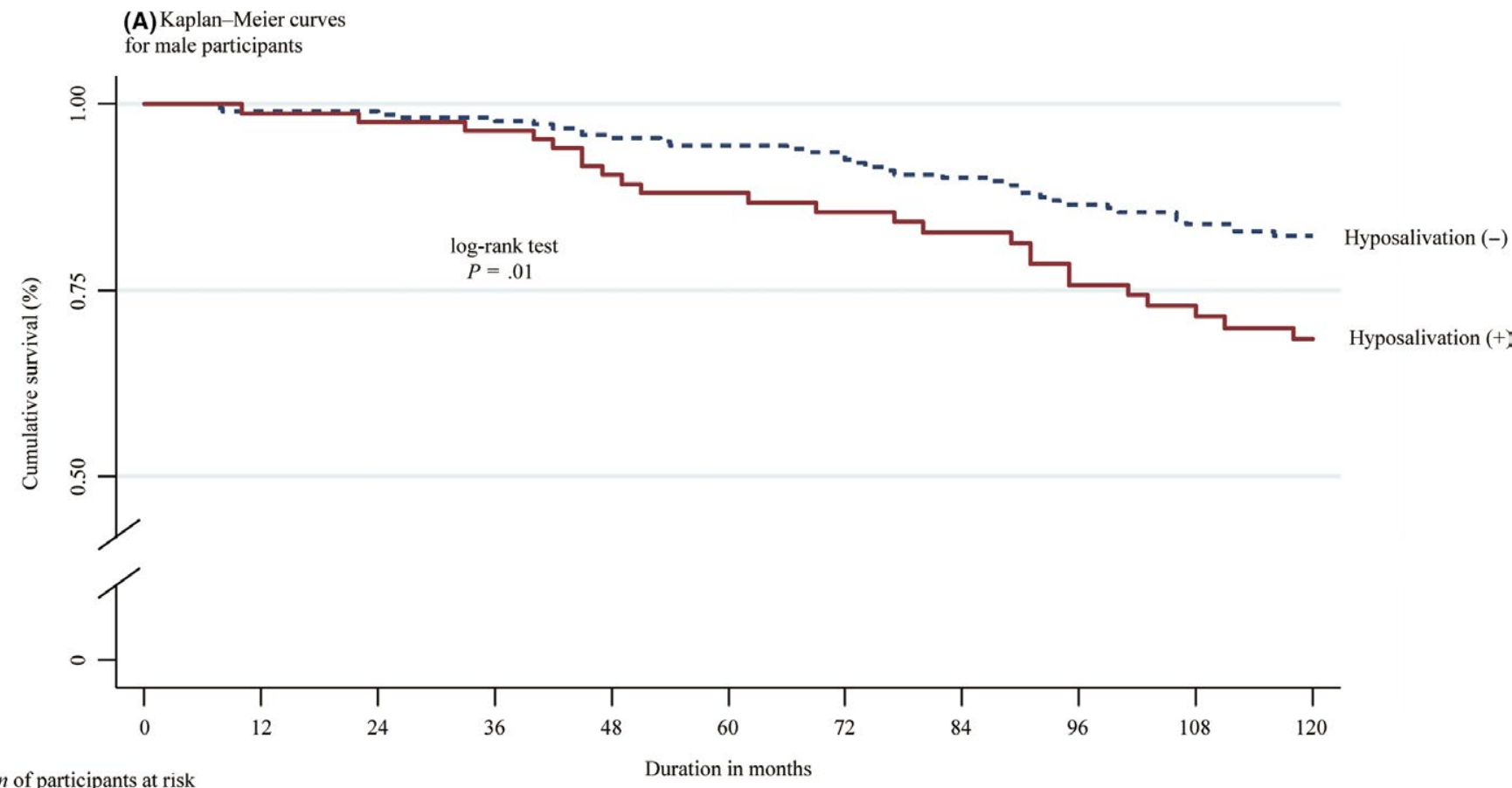

$n$ of participants at risk

Hyposalivation

\begin{tabular}{|c|c|c|c|c|c|c|c|c|c|c|}
\hline 221 & 218 & 217 & 215 & 210 & 198 & 196 & 183 & 171 & 161 & 150 \\
\hline 85 & 84 & 83 & 82 & 77 & 69 & 67 & 61 & 54 & 51 & 45 \\
\hline
\end{tabular}

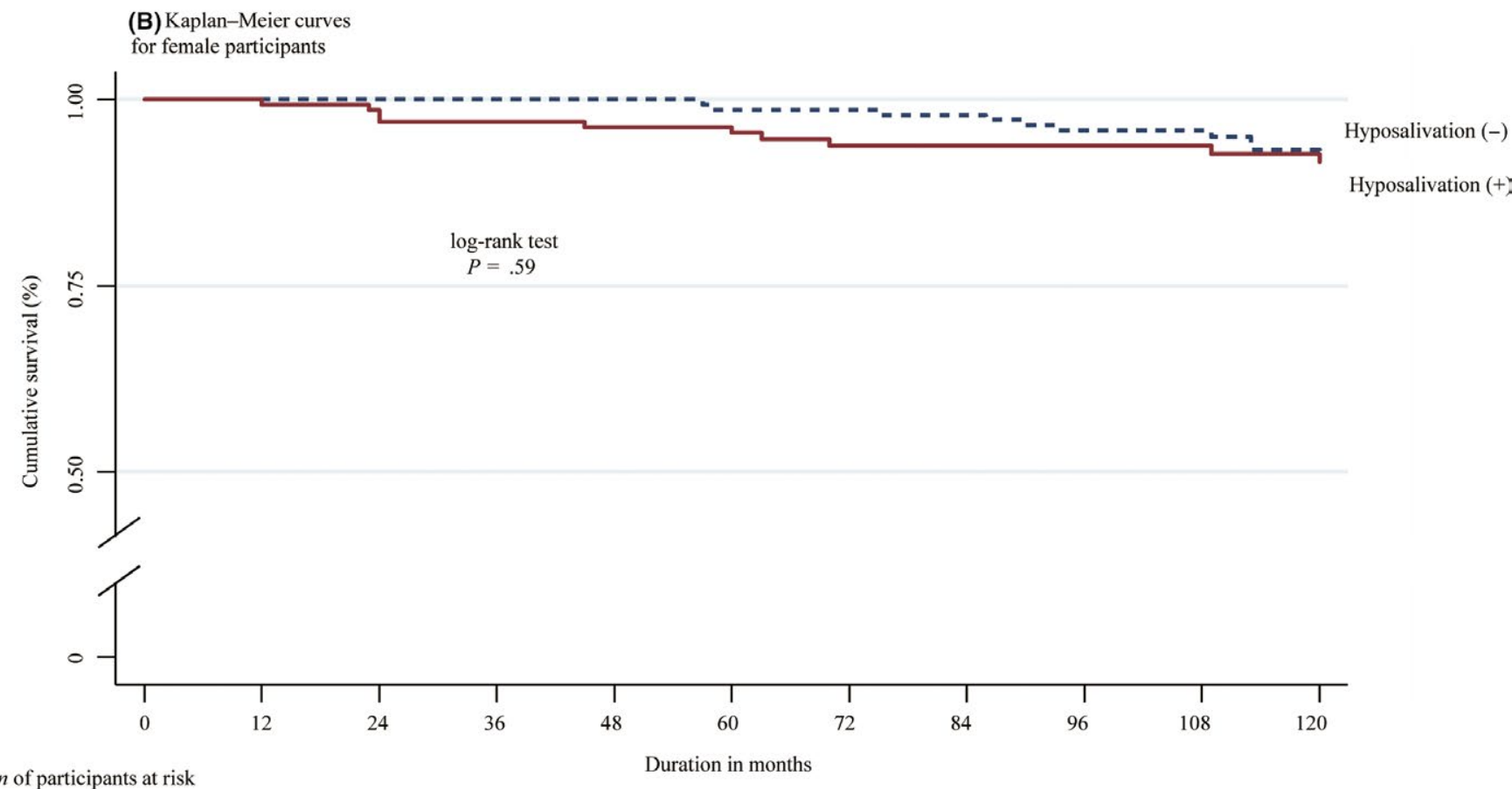

$n$ of participants at risk

Hyposalivation

\begin{tabular}{l}
- \\
\hline
\end{tabular}

FIGURE 1 Kaplan-Meier curve analysis of hyposalivation and all-cause mortality in male participants (A) and female participants (B) [Colour figure can be viewed at wileyonlinelibrary.com]

with larger sample sizes or longer observation periods are needed to draw conclusions.

In this study, the unstimulated salivary flow rate (USFR) was not measured because it often takes longer to measure (eg,
10 minutes for collection ${ }^{16}$ ) than SSFR. This factor is disadvantageous for an epidemiological survey of a large population. ${ }^{2}$ In fact, several previous epidemiological studies ${ }^{1,2,6,11,13}$ only measured the SSFR. The SSFR contributes $80 \%-90 \%$ of the average daily saliva 


\begin{tabular}{|llll|} 
& Men & Women \\
& $\mathrm{n}=306$ & $\mathrm{n}=294$ \\
\hline Predictor & $\mathrm{HR}(95 \% \mathrm{Cl})$ & $\mathrm{HR}(95 \% \mathrm{Cl})$ \\
\hline No. of teeth & $0.97(0.94-0.99)$ & $0.93(0.88-0.99)$ \\
\hline Denture use (yes vs no) & $1.19(0.71-2.00)$ & $0.67(0.28-1.61)$ \\
\hline Periodontal disease (yes vs no) & $1.08(0.57-2.11)$ & $1.14(0.44-2.98)$ \\
\hline Annual household income $<2$ 000 000 JPY (yes vs no) & $0.74(0.27-2.04)$ & $0.70(0.21-2.40)$ \\
\hline Length of school education $\leq 9$ y (yes vs no) & $0.72(0.42-1.24)$ & $2.04(0.81-5.11)$ \\
\hline Living alone (yes vs no) & $2.00(0.49-8.20)$ & $1.63(0.59-4.49)$ \\
\hline Current smoker (vs previous smoker/nonsmoker) & $1.81(1.09-3.00)$ & $\mathrm{n} / \mathrm{a}$ \\
\hline Current drinker (vs nondrinker) & $0.69(0.40-1.21)$ & $0.85(0.31-2.33)$ \\
\hline Involved in regular exercise (yes vs no) & $0.57(0.35-0.96)$ & $1.15(0.47-2.80)$ \\
\hline Polypharmacy (yes vs no) & $1.24(0.75-2.06)$ & $1.60(0.65-3.91)$ \\
\hline Decreased ADL (TMIG-IC below the median) (yes vs no) & $1.02(0.60-1.75)$ & $1.48(0.60-3.62)$ \\
\hline BMI (<18.5 kg/m ${ }^{2}$ vs $\geq 18.5$ kg/m²) & $1.37(0.65-2.88)$ & $1.73(0.51-5.91)$ \\
\hline Serum biomarker (yes vs no) & & \\
\hline Hypoalbuminemia & $2.79(1.45-5.38)$ & $6.01(2.17-16.63)$ \\
\hline Dyslipidemia & $1.04(0.55-1.96)$ & $0.68(0.28-1.67)$ \\
\hline Self-reported medical conditions (yes vs no) & & \\
\hline Hypertension & $0.65(0.34-1.21)$ & $1.30(0.47-3.57)$ \\
\hline Diabetes & $2.26(1.03-4.98)$ & $2.43(0.56-10.49)$ \\
\hline CVD & $1.93(1.06-3.52)$ & $3.44(1.01-11.77)$ \\
\hline & & \\
\hline
\end{tabular}

TABLE 2 Univariable Cox proportional hazard analyses for all-cause mortality, stratified by sex

ADL, activities of daily living; $\mathrm{BMI}$, body mass index; $\mathrm{Cl}$, confidence interval; $\mathrm{CVD}$, cardiovascular disease; HR, hazard ratio; JPY, Japanese yen; n/a, data not available because of low number; TMIG-IC, Tokyo Metropolitan Institute of Gerontology Index of Competence.

Bold text indicates statistically significant associations $(P<.05)$.

${ }^{a}$ The number of individuals analysed is 259 men and 255 women (excluding individuals with no teeth).

production. ${ }^{23}$ Previous studies have suggested that SSFR, rather than USFR, could be more strongly associated with the degree of salivary gland hypofunction. ${ }^{8,24}$ In consideration of the aforementioned fact and limited time and manpower available for the current study, we chose only to measure the SSFR. Nevertheless, we recognise the importance of future studies examining the association of USFR with mortality.

According to Screebny, ${ }^{25}$ no globally accepted definitions of hyposalivation exist for either USFR or SSFR. In this study, we defined an SSFR of $<0.7 \mathrm{~mL} / \mathrm{min}$ as hyposalivation, based on the definitions used in previous studies. ${ }^{1,16}$ The prevalence of SSFR $<0.7 \mathrm{~mL} / \mathrm{min}$ was $59.7 \%$ in a study of Mexican adults aged $\geq 60$ years, ${ }^{1}$ which is higher than the prevalence in our study (overall prevalence, 37.3\%). The difference in prevalence may be influenced by race and genetics. The study conducted in Mexico included individuals with a history of radiation therapy. This may also have affected the prevalence. One epidemiological survey of the Japanese population (mean age $=66.7$ years) reported that the prevalence of decreased SSFR (SSFR $<0.5 \mathrm{~mL}$ / min) was $20.1 \%$ and $29.6 \%$ in men and women, respectively, ${ }^{2}$ which is similar to the results obtained in the current study (the prevalence of SSFR $<0.5 \mathrm{~mL} / \mathrm{min}$ was $18.0 \%$ and $27.6 \%$ in men and women, respectively).
We chose a cut-off value of $0.7 \mathrm{~mL} / \mathrm{min}$ because this value is more commonly used in epidemiological settings. ${ }^{25}$ However, a lower cutoff value of $0.6 \mathrm{~mL} / \mathrm{min}$ was used in a previous study conducted in Japan for investigating the association between hyposalivation and the incidence of acute respiratory infection. ${ }^{11}$ Hence, we conducted additional analyses using the threshold of $0.6 \mathrm{~mL} / \mathrm{min}$ and obtained similar results for the association between hyposalivation and all-cause mortality, (aHR = 1.77 [95\% Cl, 1.02-3.09] for men; and aHR = $1.24[95 \%$ $\mathrm{Cl}, 0.51-3.04]$ for women). Because a higher cut-off value of $1.0 \mathrm{~mL} /$ min has also been used in previous studies, ${ }^{16,25}$ we repeated our analyses using this cut-off value for hyposalivation and found an aHR of allcause mortality of $1.72(95 \% \mathrm{Cl}, 1.01-2.91)$ for men and $0.99(95 \% \mathrm{Cl}$, $0.40-2.43$ ) for women, which is consistent with the aforementioned findings.

In the present study, we did not have information on the cause of death, which precluded further investigation. In addition, all-cause mortality includes potentially unrelated events, such as accidents. In other words, it is a non-specific outcome and there is thus a need for further studies investigating the associations between SSFR and cause-specific mortality. Previous studies report, for instance, that hyposalivation is associated with respiratory infections. ${ }^{11,13}$ Therefore, future studies investigating the association between SSFR and 
TAB LE 3 Crude and adjusted hazard ratios for mortality, stratified by sex

\begin{tabular}{|c|c|c|c|c|c|}
\hline Predictor & \multicolumn{5}{|l|}{ Men } \\
\hline$(-)$ & 221 & 36 & 23222 & Reference & Reference \\
\hline$(+)$ & 85 & 24 & 8238 & $1.94(1.16-3.25)$ & $1.71(1.01-2.89)$ \\
\hline High tertile (SSFR $>1.47 \mathrm{~mL} / \mathrm{min}$ ) & 94 & 11 & 10166 & Reference & Reference \\
\hline $\begin{array}{l}\text { Middle tertile }(0.80 \mathrm{~mL} / \\
\min <\mathrm{SSFR} \leq 1.47 \mathrm{~mL} / \mathrm{min})\end{array}$ & 104 & 20 & 10679 & $1.76(0.84-3.68)$ & $1.60(0.76-3.35)$ \\
\hline \multirow[t]{2}{*}{ Low tertile $(\mathrm{SSFR} \leq 0.80 \mathrm{~mL} / \mathrm{min})$} & 108 & 29 & 10615 & $2.61(1.30-5.23)$ & $2.05(1.01-4.17)$ \\
\hline & & & & $P$ for trend $=.005$ & $P$ for trend $=.045$ \\
\hline \multicolumn{6}{|l|}{ Hyposalivation } \\
\hline$(-)$ & 155 & 10 & 17210 & Reference & Reference \\
\hline$(+)$ & 139 & 10 & 13859 & $1.27(0.53-3.06)$ & $1.17(0.48-2.83)$ \\
\hline \multicolumn{6}{|l|}{ SSFR tertile category } \\
\hline High tertile (SSFR > $1.07 \mathrm{~mL} / \mathrm{min}$ ) & 88 & 6 & 9872 & Reference & Reference \\
\hline $\begin{array}{l}\text { Middle tertile }(0.60 \mathrm{~mL} / \\
\min <\mathrm{SSFR} \leq 1.07 \mathrm{~mL} / \mathrm{min})\end{array}$ & 92 & 5 & 9714 & $0.87(0.26-2.85)$ & $0.90(0.27-2.97)$ \\
\hline Low tertile (SSFR $\leq 0.60 \mathrm{~mL} / \mathrm{min})$ & 114 & 9 & 11483 & $1.32(0.47-3.72)$ & $1.18(0.42-3.36)$ \\
\hline
\end{tabular}

$\mathrm{Cl}$, confidence interval; HR, hazard ratio; PY, person-year; SSFR, stimulated salivary flow rate.

Bold text indicates statistically significant associations $(P<.05)$.

${ }^{a}$ Adjusted for number of teeth, smoking status, exercise, hypoalbuminemia, diabetes and cardiovascular disease.

${ }^{b}$ Adjusted for number of teeth, hypoalbuminemia and cardiovascular disease.

mortality from respiratory infections may shed light on the biological mechanisms underlying our findings in this study.

Besides the lack of information on cause of death, the present study has other limitations. First, each participant's salivary flow rate was measured only once in this study, as in previous studies. ${ }^{1,2,6,8,26}$ Circadian variations in salivary flow rates within individuals have been reported. A previous study demonstrated that the stimulated parotid and submandibular salivary flow rates of individuals showed up to a $44 \%$ variation over time. ${ }^{27}$ In the sensitivity analyses of this study, the SSFR was $>45 \%$ lower in the low tertile group than in the high tertile group. Therefore, we assumed that variability in the SSFR within an individual did not greatly affect the classification of the study participants. However, further studies with multiple measurements of SSFR are required. Second, this study did not include detailed information on the types and exact numbers of prescribed medications. Several types of commonly used medications, such as anticholinergic agents, antihypertensives, antidepressants and antianxiety drugs, can cause hyposalivation, ${ }^{4}$ and taking multiple medications is also associated with hyposalivation. ${ }^{16}$ In fact, we found that taking two or more medications was associated with hyposalivation in female participants. Although this study did not aim to investigate the cause of hyposalivation, these prescribed medications can reflect the participants' medical conditions and that in turn may affect mortality. In this study population, we found that taking two or more medications was not associated with an increased mortality rate. Although there is no standard cut-off point with regard to the number of medications that defines polypharmacy, five or more is a commonly agreed upon as the threshold. ${ }^{28}$ Unfortunately, we could not use that definition based on our available data. Further investigation with detailed information on the numbers and types of medications individuals were prescribed is required. Third, residual confounding remains possible because other health characteristics related to salivary output and mortality, such as cancer, depression and anxiety, ${ }^{29,30}$ was not examined and therefore not evaluated in the analyses. It may be possible that individuals with hyposalivation were more likely to have these and other medical problems. Therefore, there is a possibility that these known but unmeasured factors may confound parts of the current association. Lastly, all participants were Japanese men and women aged 70 years at enrolment, and therefore, the generalisability of the present findings is limited. 
In summary, greater all-cause mortality was observed in men with hyposalivation, but not in women. Hyposalivation could be a marker for mortality in older men. Future studies investigating the association between SSFR and cause-specific mortality are warranted.

\section{ACKNOWLEDGEMENTS}

The authors wish to thank all the Niigata study participants for their time and generosity.

\section{CONFLICT OF INTEREST}

The authors have no conflicts to declare.

\section{ORCID}

Masanori Iwasaki (iD http://orcid.org/0000-0002-5739-2936

Wenche S. Borgnakke (iD http://orcid.org/0000-0003-3593-093X

Akihiro Yoshihara (iD http://orcid.org/0000-0002-0424-8228

Kayoko Ito iD http://orcid.org/0000-0002-7511-3945

\section{REFERENCES}

1. Islas-Granillo H, Borges-Yanez A, Fernandez-Barrera MA, et al. Relationship of hyposalivation and xerostomia in Mexican elderly with socioeconomic, sociodemographic and dental factors. Sci Rep. 2017;7:40686.

2. Ikebe K, Sajima $\mathrm{H}$, Kobayashi $\mathrm{S}$, et al. Association of salivary flow rate with oral function in a sample of community-dwelling older adults in Japan. Oral Surg Oral Med Oral Pathol Oral Radiol Endod. 2002;94:184-190.

3. Ohara $\mathrm{Y}$, Hirano $\mathrm{H}$, Yoshida $\mathrm{H}$, et al. Prevalence and factors associated with xerostomia and hyposalivation among community-dwelling older people in Japan. Gerodontology. 2016;33:20-27.

4. Affoo RH, Foley N, Garrick R, Siqueira WL, Martin RE. Meta-analysis of salivary flow rates in young and older adults. J Am Geriatr Soc. 2015;63:2142-2151.

5. Mese H, Matsuo R. Salivary secretion, taste and hyposalivation. J Oral Rehabil. 2007;34:711-723.

6. Ikebe K, Matsuda K, Kagawa R, et al. Association of masticatory performance with age, gender, number of teeth, occlusal force and salivary flow in Japanese older adults: is ageing a risk factor for masticatory dysfunction? Arch Oral Biol. 2011;56:991-996.

7. Iwasaki M, Yoshihara A, Ito K, et al. Hyposalivation and dietary nutrient intake among community-based older Japanese. Geriatr Gerontol Int. 2016;16:500-507.

8. Samnieng P, Ueno M, Shinada K, Zaitsu T, Wright FA, Kawaguchi Y. Association of hyposalivation with oral function, nutrition and oral health in community-dwelling elderly Thai. Community Dent Health. 2012;29:117-123.

9. Dormenval $\mathrm{V}$, Budtz-Jorgensen $\mathrm{E}$, Mojon $\mathrm{P}$, Bruyere $\mathrm{A}$, Rapin $\mathrm{CH}$. Nutrition, general health status and oral health status in hospitalised elders. Gerodontology. 1995;12:73-80.

10. Dormenval V, Budtz-Jorgensen E, Mojon P, Bruyere A, Rapin $\mathrm{CH}$. Associations between malnutrition, poor general health and oral dryness in hospitalized elderly patients. Age Ageing. 1998;27:123-128.

11. I wabuchi H, Fujibayashi T, Yamane GY, Imai H, Nakao H. Relationship between hyposalivation and acute respiratory infection in dental outpatients. Gerontology. 2012;58:205-211.
12. Scannapieco FA, Cantos A. Oral inflammation and infection, and chronic medical diseases: implications for the elderly. Periodontol 2000. 2016;72:153-175.

13. Terpenning $M$, Bretz W, Lopatin D, Langmore S, Dominguez B, Loesche W. Bacterial colonization of saliva and plaque in the elderly. Clin Infect Dis. 1993;16(Suppl 4):S314-S316.

14. Hirotomi T, Yoshihara A, Yano M, Ando Y, Miyazaki H. Longitudinal study on periodontal conditions in healthy elderly people in Japan. Community Dent Oral Epidemiol. 2002;30:409-417.

15. Hirotomi T, Yoshihara A, Ogawa H, Ito K, Igarashi A, Miyazaki H. A preliminary study on the relationship between stimulated saliva and periodontal conditions in community-dwelling elderly people. J Dent. 2006;34:692-698.

16. Flink H, Bergdahl M, Tegelberg A, Rosenblad A, Lagerlof F. Prevalence of hyposalivation in relation to general health, body mass index and remaining teeth in different age groups of adults. Community Dent Oral Epidemiol. 2008;36:523-531.

17. Offenbacher S, Barros SP, Singer RE, Moss K, Williams RC, Beck JD. Periodontal disease at the biofilm-gingival interface. J Periodontol. 2007;78:1911-1925.

18. Murakami K, Sasaki S, Takahashi Y, et al. Hardness (difficulty of chewing) of the habitual diet in relation to body mass index and waist circumference in free-living Japanese women aged 18-22 y. Am J Clin Nutr. 2007;86:206-213.

19. The Ministry of Health, Labour and Welfare. Abridged Life Tables for Japan 2015. http://www.mhlw.go.jp/english/database/db-hw/ lifetb15/index.html. Accessed May 10, 2017.

20. von Bultzingslowen I, Sollecito TP, Fox PC, et al. Salivary dysfunction associated with systemic diseases: systematic review and clinical management recommendations. Oral Surg Oral Med Oral Pathol Oral Radiol Endod. 2007;103(Suppl):S57. e1-e15.

21. Khan GJ, Mehmood R, Salah ud D, Ihtesham ul H. Effects of long-term use of tobacco on taste receptors and salivary secretion. J Ayub Med Coll Abbottabad. 2003;15:37-39.

22. Rad M, Kakoie S, Niliye Brojeni F, Pourdamghan N. Effect of long-term smoking on whole-mouth salivary flow rate and oral health. J Dent Res Dent Clin Dent Prospects. 2010;4:110-114.

23. Humphrey SP, Williamson RT. A review of saliva: normal composition, flow, and function. J Prosthet Dent. 2001;85:162-169.

24. Valdez IH, Fox PC. Diagnosis and management of salivary dysfunction. Crit Rev Oral Biol Med. 1993;4:271-277.

25. Sreebny LM. Saliva in health and disease: an appraisal and update. Int Dent J. 2000;50:140-161.

26. Loesche WJ, Bromberg J, Terpenning MS, et al. Xerostomia, xerogenic medications and food avoidances in selected geriatric groups. J Am Geriatr Soc. 1995;43:401-407.

27. Ghezzi EM, Lange LA, Ship JA. Determination of variation of stimulated salivary flow rates. J Dent Res. 2000;79:1874-1878.

28. Maher RL, Hanlon J, Hajjar ER. Clinical consequences of polypharmacy in elderly. Expert Opin Drug Saf. 2014;13:57-65.

29. Bergdahl M, Bergdahl J. Low unstimulated salivary flow and subjective oral dryness: association with medication, anxiety, depression, and stress. J Dent Res. 2000;79:1652-1658.

30. Dewey ME, Saz P. Dementia, cognitive impairment and mortality in persons aged 65 and over living in the community: a systematic review of the literature. Int J Geriatr Psychiatry. 2001;16:751-761.

How to cite this article: Iwasaki M, Borgnakke WS, Yoshihara A, et al. Hyposalivation and 10-year all-cause mortality in an elderly Japanese population. Gerodontology. 2018;35:87-94. https:// doi.org/10.1111/ger.12319 